UDC 517.9

A. A. Martynyuk (Inst. Mech. Nat. Acad. Sci. Ukraine, Kyiv)

\title{
STABILITY ANALYSIS OF LARGE-SCALE FUNCTIONAL DIFFERENTIAL SYSTEMS
}

\section{АНАЛІЗ СТІЙКОСТІ ВЕЛИКОМАСШТАБНИХ ФУНКЦІОНАЛЬНО-ДИФЕРЕНЦІАЛЬНИХ СИСТЕМ}

The present paper is focused on the new method of analysis of stability of solutions of large-scale functional differential system via matrix-valued functional Liapunov - Krasovskii. The stability conditions are based on information about dynamical behaviour of subsystems of the general system and properties of the functions of interconnection between them.

Запропоновано один новий метод аналізу стійкості розв'язків великомасштабної функціонально-диференціальної системи на основі матричнозначного функціонала Ляпунова - Красовського. Умови стійкості грунтуються на динамічній поведінці підсистем загальної системи та властивостях функцій зв' язку між ними.

1. Introduction. Stability of continuous systems of ordinary differential equations has been studied by many authors for more that 100 years while stability investigation of solutions to functional differential equations has been undertaken quite recently. It is known (see, for example, [2, 12, 23]) that there are two global approaches of qualitative investigation of the dynamical behavior of solutions to systems of this class. One is the method of Liapunov - Razumikhin functions and the other is the method of Liapunov - Krasovskii functionals. Each approach is being intensively developed and new interesting results are obtained in both directions (see [1, 3-11, 13, 14, $22-24$, 26]).

The method of matrix-valued Liapunov functions worked out for the last years in qualitative stability theory of nonlinear systems (see [16, 17]) allows generalization investigation of solutions to functional differential equations in the framework of both of the approaches mentioned above.

In this paper, stability analysis of large-scale systems of functional differential equations with finite delay via the matrix-valued functionals of Liapunov - Krasovskii are considered. A new theorem on uniform asymptotic stability is established. These results generalize some theorems in the stability theory of functional differential equations via scalar Liapunov functionals obtained recently.

The results of this paper are arranged as follows.

We provide a statement the problem in Section 2 and provide necessary information on functional differential equations and matrix-valued Liapunov - Krasovskii functionals in Section 3.

In Section 4 we elaborate on the various types of qualitative properties (i.e., stability properties) of large-scale functional differential equations that we will consider. In Section 5 we specialize the results of Section 4 for a class of functional differential equations and proposes new forms of decomposition-aggregation of largescale systems of functional differential equations via the application of matrix-valued functionals. This follows us to establish new sufficient stability conditions for the systems of equations under consideration in terms of special matrices property of fixed sign.

2. Statement the problem. For $x \in R^{n},|\cdot|$ denotes the Euclidean norm of $x$. Let $C=C\left([-\tau, 0], R^{n}\right)$ be the space of continuous functions which map $[-\tau, 0]$ into $R^{n}$ and for $\varphi \in C,\|\varphi\| \leq \sup _{-\tau \leq \theta \leq 0}|\varphi(\theta)|, C_{H}$ is a set $\varphi \in C$ for which $\|\varphi\|<H$, and $x_{t}$, as an element of $C$, is defined by correlation $x_{t}(\theta)=x(t+\theta),-\tau \leq \theta \leq 0$.

Throughout this paper we will use a seminorm $|\cdot|_{\tau}$ on $C$ with $|\varphi|_{\tau} \leq\|\varphi\|$ for all $\varphi \in C$.

We consider the large-scale systems described by functional differential equations 


$$
\frac{d x}{d t}=f\left(t, x_{t}\right), \quad x_{t_{0}}=\varphi_{0} \in C, \quad t_{0} \geq 0
$$

where $f \in C\left(R_{+} \times C, R^{n}\right), x \in C\left(\left[t_{0}-\tau, \infty\right], R^{n}\right)$, and $x_{t} \in C$. In $(2.1), d x / d t$ denotes the right-hand derivative of $x$ at $x \in R_{+}$. We suppose that for every $\varphi \in C$ and for every $t_{0} \geq 0$, system (2.1) possesses a unique solution $x_{t}\left(t_{0}, \varphi\right)$ with $x_{t_{0}}=\varphi$ and we denote by $x(t)=x\left(t ; t_{0}, \varphi\right)$ the value of $x_{t}\left(t_{0}, \varphi\right)$ at $t$ (for details see [2]).

Let the system (2.1) be decomposed into $m$ interconnected subsystems described by the equations

$$
\frac{d x^{i}}{d t}=f_{i}\left(t, x_{t}^{i}\right)+g_{i}\left(t, x_{t}^{1}, \ldots, x_{t}^{m}\right)
$$

where $i \in I_{m}=\{1,2, \ldots, m\}, \quad \sum_{i=1}^{m} n_{i}=n, f_{i} \in C\left(R_{+} \times C_{H_{i}}, R^{n_{i}}\right), \quad$ and $g_{i} \in$ $\in C\left(R_{+} \times C_{H}, R^{n_{i}}\right), C_{H}=C_{H_{1}} \times \ldots \times C_{H_{m}}$, i.e., $C_{H_{i}}$ is a set of $\varphi_{i} \in C$ for which $\left\|\varphi^{i}\right\|<H_{i}, i \in I_{m}$. When $g_{i}\left(t, x_{t}\right) \equiv 0$ for all $i \in I_{m}$, from (2.2) we obtain the isolated subsystems

$$
\frac{d x^{i}}{d t}=f_{i}\left(t, x_{t}^{i}\right), \quad x_{t_{0}}^{i} \in \varphi_{0}^{i} \in C_{H_{i}}, \quad t_{0} \geq 0 .
$$

We also assume that $f(t, 0, \ldots, 0)=0, f_{i}(t, 0)=0$, and $g_{i}(t, 0, \ldots, 0)=0$ for all $i \in I_{m}$, so that $x=0\left(x^{i}=0\right)$ are the solutions of (2.1) or (2.) respectively.

3. Auxiliary results. We will need some notions which we present now.

Definition 3.1. The functional $U(t, \varphi)=\left[v_{i j}(t, \cdot)\right], i, j=1,2, \ldots, m$, continuous and defined on $R_{+} \times C_{H}$ which together with the upper right Dini derivative of $V(t, \varphi, \eta)=\eta^{T} U(t, \varphi) \eta, \eta \in R_{+}^{m}$, defined by

$$
\begin{gathered}
\left.D^{+} V(t, \varphi, \eta)\right|_{(2.1)}= \\
=\limsup \left\{\left[V\left(t+h, x_{t+h}\left(t_{0}, \varphi\right), \eta\right)-V\left(t, x_{t}\left(t_{0}, \varphi\right), \eta\right)\right] h^{-1}: h \rightarrow 0^{+}\right\},
\end{gathered}
$$

solves the problem of stability of solution $x=0$, is called Liapunov matrix-valued functional.

Definition 3.2. The function $w:[0, \infty) \rightarrow[0, \infty), w(0)=0, w(r)$ strictly increasing with $w(0)=0$ and $w(r) \rightarrow \infty$ for $r \rightarrow+\infty$, is called a wedge function (for short we will use $w \in W$ ).

Definition 3.3. The zero solution of (2.1) is

i) stable if for each $\varepsilon>0$ there exists $\delta=\delta\left(t_{0}, \varepsilon\right)$ such that $\left[\left(t_{0}, \varphi\right) \in\right.$ $\left.\in R_{+} \times C_{H},\|\varphi\|<\delta, t \geq t_{0}\right]$ implies $\left|x\left(t ; t_{0}, \varphi\right)\right|<\varepsilon$ for all $t \geq t_{0}$;

ii) uniformly stable if it is stable and if in the Definition 2.3(a) the value $\delta$ does not depend on $t_{0}$;

iii) asymptotically stable $(A S)$ if it is stable and for any $t_{0} \geq 0$ there exists $\Delta>$ $>0$ such that $\left[(t, \varphi) \in R_{+} \times C_{H},\|\varphi\|<\Delta, t \geq t_{0}\right]$ implies $\left|x\left(t ; t_{0}, \varphi\right)\right| \rightarrow 0$ for $t \rightarrow+\infty$;

iv) uniformly asymptotically stable (UAS) if it is uniformly stable and there exists $\delta^{*}>0$ such that for each $\varepsilon>0$ there exists $T>0$ such that $\left[\left(t_{0}, \varphi\right) \in R_{+} \times C_{H}\right.$, $\left.\|\varphi\|<\delta^{*}, t_{0} \geq 0\right]$ implies $\left|x\left(t ; t_{0}, \varphi\right)\right|<\varepsilon$ for all $t \geq t_{0}+T$. 
4. Stability of large-scale system. It is known that the problem of constructing appropriate Liapunov - Krasovskii functionals which solve the stability problem of solution $x=0$ of system (2.1) is a central one in the direct Liapunov method for functional differential equations (2.1). The application of matrix-valued functionals allows one to extend the class of admissible components for construction of the appropriate Liapunov - Krasovskii functional.

Further we shall investigate systems of connected equations (2.2) and independent subsystems (2.3) in the framework of general methodology of qualitative analysis of large-scale systems motion.

4.1. Approach $\boldsymbol{A}_{1}$. This approach is based on the system of conditions below.

Assumption 4.1. There exist functionals

$$
v_{i i}\left(t, \varphi^{i}\right): R_{+} \times C_{H_{i}} \rightarrow R_{+} \text {and } v_{i j}\left(t, \varphi^{i}, \varphi^{j}\right): R_{+} \times C_{H_{i}} \times C_{H_{j}} \rightarrow R,
$$

wedge functions $\tilde{w}_{1}, \quad \tilde{w}_{2}, \quad \tilde{w}_{3} \in W$-class and constants $\tilde{a}_{i j}, \quad \tilde{b}_{i j}, \quad \tilde{c}_{i j}, i, j=1,2, \ldots$ $\ldots, m$, and $0<K_{i}<H$ such that

1) $\tilde{a}_{i i} \tilde{w}_{i 1}^{2}\left(\left|\varphi^{i}(0)\right|\right) \leq v_{i i}\left(t, \varphi^{i}\right) \leq \tilde{b}_{i i} \tilde{w}_{i 2}^{2}\left(\left|\varphi^{i}\right|_{\tau}\right)+\tilde{c}_{i i} \tilde{w}_{i 3}^{2}\left(\left\|\varphi^{i}\right\|\right)$ for all $(t$, $\left.\varphi^{i}\right) \in R_{+} \times C_{K_{i}}, \quad i=1,2, \ldots, m$;

2) $\tilde{a}_{i j} \tilde{w}_{i 1}\left(\left|\varphi^{i}(0)\right|\right) \tilde{w}_{j 1}\left(\left|\varphi^{j}(0)\right|\right) \leq v_{i j}\left(t, \varphi^{i}, \varphi^{j}\right) \leq \tilde{b}_{i j} \tilde{w}_{i 2}\left(\left|\varphi^{i}\right|_{\tau}\right) \tilde{w}_{j 2}\left(\left|\varphi^{j}\right|_{\tau}\right)+$ $+\tilde{c}_{i j} \tilde{w}_{i 3}\left(\left\|\varphi^{i}\right\|\right) \tilde{w}_{j 3}\left(\left\|\varphi^{j}\right\|\right)$ for all $\left(t, \varphi^{i}, \varphi^{j}\right) \in R_{+} \times C_{K_{i}} \times C_{K_{j}}, i \neq j \in I_{m}$.

The following assertion holds true.

Proposition 4.1. If all conditions of Assumption 4.1 are satisfied, then in the domain of values $(t, \varphi) \in R_{+} \times C_{K}$ for the functional

$$
V(t, \varphi, \eta)=\eta^{T} U(t, \varphi) \eta, \quad \eta \in R_{+}^{m}, \quad \eta>0,
$$

the bilateral inequality

$$
\begin{gathered}
\tilde{w}_{1}^{T}(|\varphi(0)|) H^{T} \tilde{A} H \tilde{w}_{1}(|\varphi(0)|) \leq V(t, \varphi, \eta) \leq \\
\leq \tilde{w}_{2}^{T}\left(|\varphi|_{\tau}\right) H^{T} \tilde{B} H \tilde{w}_{2}\left(|\varphi|_{\tau}\right)+\tilde{w}_{3}^{T}(\|\varphi\|) H^{T} \tilde{C} H \tilde{w}_{3}(\|\varphi\|)
\end{gathered}
$$

is fulfilled for all $(t, \varphi) \in R_{+} \times C_{K}$, where

$$
\begin{gathered}
\tilde{w}_{1}^{T}(|\varphi(0)|)=\left(\tilde{w}_{11}\left(\left|\varphi^{1}(0)\right|\right), \ldots, \tilde{w}_{m 1}\left(\left|\varphi^{m}(0)\right|\right)\right), \\
\tilde{w}_{2}^{T}\left(|\varphi|_{\tau}\right)=\left(\tilde{w}_{12}\left(\left|\varphi^{1}\right|_{\tau}\right), \ldots, \tilde{w}_{m 2}\left(\left|\varphi^{m}\right|_{\tau}\right)\right), \\
\tilde{w}_{3}^{T}(\|\varphi\|)=\left(\tilde{w}_{13}\left(\left\|\varphi^{1}\right\|\right), \ldots, \tilde{w}_{m 3}\left(\left\|\varphi^{m}\right\|\right)\right), \\
\tilde{A}=\left[\tilde{a}_{i j}\right], \quad \tilde{a}_{i j}=\tilde{a}_{j i}, \quad \tilde{B}=\left[\tilde{b}_{i j}\right], \quad \tilde{b}_{i j}=\tilde{b}_{j i}, \\
\tilde{C}=\left[\tilde{c}_{i j}\right], \quad \tilde{c}_{i j}=\tilde{c}_{j i}, \quad H=\operatorname{diag}\left[\eta_{1}, \ldots, \eta_{m}\right], \\
C_{K}=C_{K_{1}} \times C_{K_{2}} \times \ldots \times C_{K_{m}} .
\end{gathered}
$$

Proposition 4.1 is proved by direct substitution by estimates 1), 2) of Assumption 4.1 in the expression of functional

$$
V(t, \varphi, \eta)=\sum_{i, j=1}^{m} \eta_{i} \eta_{j} v_{i j}(t, \cdot)
$$

with subsequent simple transformations. 
Assumption 4.2. There exist functionals $v_{i i}\left(t, \varphi^{i}\right), i=1,2, \ldots, m$, and $v_{i j}(t$, $\left.\varphi^{i}, \varphi^{j}\right), i \neq j \in I_{m}$, mentioned in Assumption 4.1 which are locally Lipschitzian in $\varphi^{i}$ and $\left(\varphi^{i}, \varphi^{j}\right)$ respectively, wedge functions $\tilde{w}_{i 4}, i=1,2, \ldots, m$, and continuous positive function $d_{k i}(t), d_{k i j}(t): R_{+} \rightarrow R_{+}, k=1,2,3, i, j=1,2, \ldots, m$, $i \neq j$, such that

1) $\left.D^{+} v_{i i}\left(t, \varphi^{i}\right)\right|_{(2.3)} \leq d_{1 i} \tilde{w}_{i 4}^{2}\left(\left|\varphi^{i}\right|_{\tau}\right)$ for all $\left(t, \varphi^{i}\right) \in R_{+} \times C_{K_{i}}$;

2) $\left.D^{+} v_{i i}\left(t, \varphi^{i}\right)\right|_{(2.2)}-\left.D^{+} v_{i i}\left(t, \varphi^{i}\right)\right|_{(2.3)} \leq \sum_{\substack{i=1 \\ i \neq j}}^{m} d_{1 i j} \tilde{w}_{i 4}\left(\left|\varphi^{i}\right|_{\tau}\right) \tilde{w}_{j 4}\left(\left|\varphi^{j}\right|_{\tau}\right)$

for all $\left(t, \varphi^{i}, \varphi^{j}\right) \in R_{+} \times C_{K_{i}} \times C_{K_{j}}, \quad i \neq j ;$

3) $\left.D^{+} v_{i j}\left(t, \varphi^{i}, \varphi^{j}\right)\right|_{(2.2)} \leq d_{2 i} \tilde{w}_{i 4}^{2}\left(\left|\varphi^{i}\right|_{\tau}\right) \leq$

$\leq \sum_{\substack{i=1 \\ i \neq j}}^{m} d_{2 i j} \tilde{w}_{i 4}\left(\left|\varphi^{i}\right|_{\tau}\right) \tilde{w}_{j 4}\left(\left|\varphi^{j}\right|_{\tau}\right)+\sum_{\substack{i=1 \\ i \neq j}}^{m} d_{3 i j} \tilde{w}_{i 4}\left(\left|\varphi^{i}\right|_{\tau}\right) \tilde{w}_{j 4}\left(\left|\varphi^{j}\right|_{\tau}\right)+d_{3 i} \tilde{w}_{j 4}^{2}\left(\left|\varphi^{j}\right|_{\tau}\right)$

for all $\left(t, \varphi^{i}, \varphi^{j}\right) \in R_{+} \times C_{K_{i}} \times C_{K_{j}}, \quad i \neq j$.

Proposition 4.2. If all conditions of Assumption 4.2 are satisfied, then

$$
\left.D^{+} V(t, \varphi, \eta)\right|_{(2.2)} \leq \tilde{w}_{4}^{T}\left(|\varphi|_{\tau}\right) D \tilde{w}_{4}\left(|\varphi|_{\tau}\right)
$$

for all $(t, \varphi) \in R_{+} \times C_{K}, C_{K}=C_{K_{1}} \times C_{K_{2}} \times \ldots \times C_{K_{m}}$, where

$$
\begin{gathered}
\tilde{w}_{4}\left(|\varphi|_{\tau}\right)=\left(\tilde{w}_{14}\left(\left|\varphi^{1}\right|_{\tau}\right), \ldots, \tilde{w}_{m 4}\left(\left|\varphi^{m}\right|_{\tau}\right)\right)^{T}, \\
D(t)=\left[d_{i j}(t)\right], \quad d_{i j}=d_{j i}, \quad i, j=1,2, \ldots, m, \\
d_{i i}(t)=\eta_{i}^{2} d_{1 i}+2 \sum_{\substack{j=2 \\
j>i}}^{m} \eta_{i} \eta_{j}\left(d_{2 i}+d_{3 j}\right), \quad i=1,2, \ldots, m, \\
d_{i j}(t)=\frac{1}{2} \eta_{i}^{2}\left(d_{1 i j}(t)+d_{1 j i}(t)\right)+\eta_{i} \eta_{j}\left[\sum_{\substack{s=1 \\
s \neq j}}^{m} d_{2 s j}(t)+\sum_{\substack{s=1 \\
s \neq j}}^{m} d_{3 s j}(t)\right], \quad(i \neq j) \in I_{m} .
\end{gathered}
$$

Proof. Estimate (4.3) is obtained by direct substitution by estimates 1) - 3) from Assumption 4.2 in the expression

$$
D^{+} V(t, \varphi, \eta)=\eta^{T} D^{+} U(t, \varphi) \eta, \quad \eta \in R_{+}^{m},
$$

where $D^{+} U(t, \varphi)$ is computed component-wise along solutions of subsystems (2.3) and (2.2) respectively.

Estimates (4.2), (4.3) and Theorem 4.1 of the paper [15] enable us to establish new stability conditions for the solution $x=0$ of system (2.1) as follows.

Theorem 4.1. Assume that for system (2.1) the functional $U(t, \varphi)$, is constructed for the components of which all estimates of Assumption 4.1 are fulfilled and for the upper right derivatives $D^{+} v_{i j}(t, \cdot)$ estimates 1) - 3) of Assumption 4.2 are fulfilled, and moreover 
a) in estimates (4.2) matrices $A=H^{T} \tilde{A} H, B=H^{T} \tilde{B} H$ and $C=H^{T} \tilde{C} H$ are positive definite;

b) there exist $r_{0} \leq \min _{i} K_{i}$ such that $\lambda_{m}(A) w_{1}(r)-\lambda_{M}(C) w_{3}(r)>0$ for all $r \in\left(0, r_{0}\right)$, where $w_{1}, w_{2}$ are the wedge functions such that $w_{1}(r) \leq \tilde{w}_{1}^{T}(r) \tilde{w}_{1}(r)$, $w_{3}(r) \geq \tilde{w}_{3}^{T}(r) \tilde{w}_{3}(r)$ for any $r \in\left(0, r_{0}\right)$.

Then the zero solution of system (2.1) is

a) uniformly stable, if the constant matrix $D_{M} \geq \frac{1}{2}\left(D^{T}(t)+D(t)\right)$ in inequality (4.3) is negative semidefinite;

b) uniformly asymptotically stable if the matrix $D_{M}$ mentioned in condition a) is negative definite.

Proof. Under condition a) of Theorem 4.1 estimate (4.2) implies that the functional (4.1) is positive definite and decreasing and the upper right derivative of the functional $V(t, \varphi, \eta)$ satisfies the condition

$$
\left.D^{+} V(t, \varphi, \eta)\right|_{(2.1)} \leq 0 \text { for all }(t, \varphi) \in R_{+} \times C_{K} .
$$

Therefore all conditions of Theorem 4.1(2) of the paper [15] are satisfied and the zero solution of system (2.1) is uniformly stable. Assertion b) of this theorem is proved in the same way in view of Theorem 4.1(3) of the same paper [15].

4.2. Approach $\boldsymbol{A}_{2}$. System of conditions of Assumption 4.2 outlines a general approach to stability analysis of zero solution of system (2.1). The essence of this approach is that stability conditions of zero solution of system (2.1) are established based on the analysis of dynamical properties of independent subsystems (4.3) and qualitative estimates of interconnection functions between them. Below the other system of conditions is presented under which stability of system (2.1) can be studied in the framework of this approach.

Assumption 4.3. There exist functionals $v_{i i}\left(t, \varphi^{i}\right)$ and $v_{i j}\left(t, \varphi^{i}, \varphi^{j}\right)$, mentioned in Assumption 4.1 wedge functions $\tilde{w}_{i 4}, i=1,2, \ldots, m$, and constants $d_{k i}, d_{i j}, k=1,2, i, j=1,2, \ldots, m$, such that

1) $\left.D^{+} v_{i i}\left(t, \varphi^{i}\right)\right|_{(2.3)} \leq d_{1 i} \tilde{w}_{i 4}^{2}\left(\left|\varphi^{i}\right|_{\tau}\right)$ for all $\left(t, \varphi^{i}\right) \in R_{+} \times C_{K_{i}}$;

2) $\sum_{i=1}^{m} \eta_{i}\left\{\left.D^{+} v_{i i}\left(t, \varphi^{i}\right)\right|_{(2.2)}-\left.D^{+} v_{i i}\left(t, \varphi^{i}\right)\right|_{(2.3)}+\right.$

$$
\begin{gathered}
\left.+\sum_{\substack{j=1 \\
j \neq i}}^{m} \eta_{j}\left(D^{+} v_{i j}\left(t, \varphi^{i}, \varphi^{j}\right)_{(2.2)}\right)\right\} \leq \sum_{i=1}^{m} d_{2 i} \tilde{w}_{i 4}^{2}\left(\left|\varphi^{i}\right|_{\tau}\right)+ \\
+\sum_{\substack{j=1 \\
j \neq i}}^{m} d_{i j} \tilde{w}_{i 4}\left(\left|\varphi^{i}\right|_{\tau}\right) \tilde{w}_{j 4}\left(\left|\varphi^{j}\right|_{\tau}\right) \text { for all }\left(t, \varphi^{i}, \varphi^{j}\right) \in R_{+} \times C_{K_{i}} \times C_{K_{j}} .
\end{gathered}
$$

Remark 4.1. In some cases condition 2) from Assumption 4.3 allows one to estimate more precisely the effect of the interconnection functions $g_{i}\left(t, x_{t}^{1}, \ldots, x_{t}^{m}\right), i=$ $=1,2, \ldots, m$, on the whole system dynamics.

Proposition 4.3. If all conditions of Assumption 4.3 are satisfied, then

$$
\left.D^{+} V(t, \varphi, \eta)\right|_{(2.2)} \leq \tilde{w}_{4}^{T}\left(|\varphi|_{\tau}\right) E \tilde{w}_{4}\left(|\varphi|_{\tau}\right)
$$


for all $(t, \varphi) \in R_{+} \times C_{K}$, where $\tilde{w}_{4}\left(|\varphi|_{\tau}\right)$ is determined as in Proposition 4.2

$$
\begin{gathered}
E=\left[\varepsilon_{i j}\right], \quad \varepsilon_{i j}=\varepsilon_{j i}, \quad i, j=1,2, \ldots, m, \\
\varepsilon_{i i}=d_{1 i}+d_{2 i}, \quad i=1,2, \ldots, m, \\
\varepsilon_{i j}=\frac{1}{2} d_{i j}, \quad i \neq j, \quad(i, j) \in I_{m} .
\end{gathered}
$$

This proposition is proved in the same way as Proposition 4.2.

Theorem 4.2. Assume that for system (2.2) the matrix-valued functional $U(t, \varphi)$ is constructed for which all estimates of Assumption 4.1 are fulfilled and for the upper right derivatives $D^{+} v_{i j}(t, \cdot)$ estimates of Assumption 4.3 and conditions a), b) of Theorem 4.1 are satisfied. Then the zero solution of system (2.1) is

a) uniformly stable, if the matrix $E$ in estimate (4.4) is negative semidefinite;

b) uniformly asymptotically stable, if the matrix $E$ in estimate (4.4) is negative definite.

The proof of Theorem 4.2 is omitted, since it is similar to the proof of Theorem 4.1.

4.3. Approach $\boldsymbol{B}_{1}$. In distinction to the system of conditions from Assumptions 4.2 and 4.3, which is the basis for Theorem 4.2 we shall indicate the approach of establishing stability conditions for zero solution of system (2.1), in which the interacting subsystem is not divided into free subsystem and interconnection functions. Besides some restrictions are imposed simultaneously on all interacting subsystems (2.1).

Assumption 4.4. There exist functionals $v_{i i}\left(t, \varphi^{i}\right)$ and $v_{i j}\left(t, \varphi^{i}, \varphi^{j}\right)$, mentioned in Assumption 4.1, wedge functions $\tilde{w}_{i 4}$ and bounded on any finite interval functions $\beta_{1 i}(t), \beta_{2 i}(t), \beta_{3 i}(t), \beta_{k i j}(t), k=1,2,3, \quad(i \neq j) \in I_{m}$, such that

1) $\left.D^{+} v_{i i}\left(t, \varphi^{i}\right)\right|_{(2.3)} \leq \beta_{1 i}(t) \tilde{w}_{i 4}^{2}\left(\left|\varphi^{i}\right|_{\tau}\right)+\sum_{j=1}^{m} \beta_{1 i j}(t) \tilde{w}_{i 4}\left(\left|\varphi^{i}\right|_{\tau}\right) \tilde{w}_{j 4}\left(\left|\varphi^{j}\right|_{\tau}\right)$

for all $\left(t, \varphi^{i}, \varphi^{j}\right) \in R_{+} \times C_{K_{i}} \times C_{K_{j}}, \quad i=1,2, \ldots, m$;

2) $\left.D^{+} v_{i j}\left(t, \varphi^{i}, \varphi^{j}\right)\right|_{(2.2)} \leq \beta_{2 i}(t) \tilde{w}_{i 4}^{2}\left(\left|\varphi^{i}\right|_{\tau}\right)+$

$$
+\sum_{\substack{j=1 \\ i \neq j}}^{m} \beta_{2 i j}(t) \tilde{w}_{i 4}\left(\left|\varphi^{i}\right|_{\tau}\right) \tilde{w}_{j 4}\left(\left|\varphi^{j}\right|_{\tau}\right)+
$$

$+\sum_{j=1}^{m} \beta_{3 i j}(t) \tilde{w}_{i 4}\left(\left|\varphi^{i}\right|_{\tau}\right) \tilde{w}_{j 4}\left(\left|\varphi^{j}\right|_{\tau}\right)+\beta_{3 i}(t) \tilde{w}_{i 4}^{2}\left(\left|\varphi^{i}\right|_{\tau}\right)$ for all $\left(t, \varphi^{i}, \varphi^{j}\right) \in R_{+} \times$ $i \neq j$

$\times C_{K_{i}} \times C_{K_{j}}, \quad(i \neq j) \in I_{m}$.

Proposition 4.4. If all conditions of Assumption 4.4 are satisfied, then

$$
\left.D^{+} V(t, \varphi, \eta)\right|_{(2.2)} \leq \tilde{w}_{4}^{T}\left(|\varphi|_{\tau}\right) \theta(t) \tilde{w}_{4}\left(|\varphi|_{\tau}\right)
$$

for all $(t, \varphi) \in R_{+} \times C_{K}$. Here the elements of matrix $\theta(t)=\left[\theta_{i j}(t)\right], \theta_{i j}=\theta_{j i}$ for all $(i, j) \in I_{m}$, are determined as: 


$$
\begin{gathered}
\theta_{i i}(t)=\eta_{i}^{2} \beta_{1 i}(t)+2 \sum_{\substack{j=2 \\
j>i}}^{m} \eta_{i} \eta_{j}\left(\beta_{2 i}(t)+\beta_{3 j}(t)\right), \quad i=1,2, \ldots, m, \\
\theta_{i j}(t)=\frac{1}{2} \eta_{i}^{2} \beta_{1 i j}(t)+\eta_{i} \eta_{j}\left(\sum_{\substack{i=1 \\
i \neq j}}^{m} \beta_{2 i j}(t)+\sum_{\substack{j=1 \\
j \neq i}}^{m} \beta_{3 i j}(t)\right), \quad(i \neq j) \in I_{m} .
\end{gathered}
$$

The proof of this assertion is similar to those of Propositions 4.2 and 4.3 and so are omitted here.

Theorem 4.3. Assume that for system (2.1) the matrix-valued functional $U(t$, $\varphi)$, is constructed for components of which all conditions of Assumption 4.1 are fulfilled and for the upper derivatives $D^{+} v_{i j}(t, \cdot)$ estimates of Assumption 4.4 are satisfied as well as conditions a) and b) from Theorem 4.1.

Then the zero solution of system (2.1):

a) uniformly stable, if the constant $m \times m$-matrix $\theta_{M} \geq \frac{1}{2}\left(\theta^{T}(t)+\theta(t)\right)$ in estimate (4.5) is negative semidefinite;

b) uniformly asymptotically stable, if the matrix $\theta_{M}$ mentioned in condition a) is negative definite.

The proof of this theorem is similar to that of Theorem 4.2.

4.4. Approach $\boldsymbol{B}_{2}$. This approach is based on the following system of conditions.

Assumption 4.5. There exist functionals $v_{i i}\left(t, \varphi^{i}\right)$ and $v_{i j}\left(t, \varphi^{i}, \varphi^{j}\right)$ for $(i \neq$ $\neq j) \in I_{m}$, mentioned in Assumption 4.1, wedgefunctions $\tilde{w}_{i 4}$ and constants $\beta_{i i}$, $i=1,2, \ldots, m, \beta_{i j},(i \neq j) \in I_{m}$, such that

$$
\begin{gathered}
\left.\sum_{i=1}^{m} \eta_{i}^{2}\left(D^{+} v_{i i}\left(t, \varphi^{i}\right)\right)\right|_{(2.2)}+\left.2 \sum_{i=1}^{m-1} \sum_{\substack{j=2 \\
j>i}}^{m} \eta_{i} \eta_{j}\left(D^{+} v_{i j}\left(t, \varphi^{i}, \varphi^{j}\right)\right)\right|_{(2.2)} \leq \\
\leq \sum_{i=1}^{m} \beta_{i i} \tilde{w}_{i 4}^{2}\left(\left|\varphi^{i}\right|_{\tau}\right)+\sum_{i=1}^{m-1} \sum_{j=2}^{m} \beta_{i j} \tilde{w}_{i 4}\left(\left|\varphi^{i}\right|_{\tau}\right) \tilde{w}_{j 4}\left(\left|\varphi^{j}\right|_{\tau}\right)
\end{gathered}
$$

for all $\left(t, \varphi^{i}, \varphi^{j}\right) \in R_{+} \times C_{K_{i}} \times C_{K_{j}}$.

Similarly to the above proposition the following assertion takes place.

Proposition 4.5. If all conditions of Assumption 4.5 are satisfied, then

$$
\left.D^{+} V(t, \varphi, \eta)\right|_{(2.2)} \leq \tilde{w}_{4}^{T}\left(|\varphi|_{\tau}\right) P \tilde{w}_{4}\left(|\varphi|_{\tau}\right)
$$

for all $(t, \varphi) \in R_{+} \times C_{K}$, where the matrix $P=\left[\rho_{i j}\right], \rho_{i j}=\rho_{j i}$ for all $(i, j) \in I_{m}$, has the elements

$$
\rho_{i j}=\beta_{i i}, \quad i=1,2, \ldots, m ; \quad \rho_{i j}=\frac{1}{2} \beta_{i j}, \quad(i \neq j) \in I_{m} .
$$

The following result establishes stability conditions for zero solution of (2.1).

Theorem 4.4. Assume that for interacting subsystems (2.2) the matrix-valued functional $U(t, \varphi)$, is constructed, for the components of which all conditions of Assumption 4.1 are satisfied and for the upper derivatives $D^{+} v_{i j}(t, \cdot)$ the estimates of Assumption 4.5 are fulfilled as well as conditions a) and b) from Theorem 4.1 . 
Then the zero solution of system (2.1) is

a) uniformly stable if the matrix $P$ in the inequality (4.6) is negative semidefinite;

b) uniformly asymptotically stable if the matrix $P$ in the inequality (4.6) is negative definite.

The proof of this theorem is similar to that of Theorem 3.1.

Remark 4.2. Theorems $4.1-4.4$ given a series of corollaries. Below a corollary of Theorem 4.1, is presented being of importance in the next section.

Corollary 4.1. Assume that for system (2.2) the matrix-valued functional $U(t$, $\varphi)$ be constructed such that the functional $U(t, \varphi, \eta)$ is continuous on $R_{+} \times C_{K}$, and locally Lipschitzian in $\varphi$ and

i) $\tilde{w}_{1}^{T}(|\varphi(0)|) A \tilde{w}_{1}(|\varphi(0)|) \leq V(t, \varphi, \eta) \leq \tilde{w}_{2}^{T}(\|\varphi\|) B \tilde{w}_{2}(\|\varphi\|)$, where $A, B$ are constant $m \times m$ matrices and $\tilde{w}_{1}, \tilde{w}_{2}$ are wedge functions;

ii) $\left.D^{+} V(t, \varphi, \eta)\right|_{(2.2)} \leq-\tilde{w}_{3}^{T}(|\varphi(0)|) D \tilde{w}_{3}(|\varphi(0)|)$ for all $(t, \varphi) \in R_{+} \times C_{K}$, where $\tilde{D}$ is a constant $m \times m$ matrix;

iii) $\lambda_{m}(A)>0, \lambda_{m}(B)>0, \lambda_{M}(\tilde{D})>0$.

Then the zero solution of system (2.2) is uniformly asymptotically stable.

5. Applications. Consider a linear delay system consisting of two interconnected subsystems. To analyse stability of its zero solution we shall apply Approach $A_{1}$. Let the system be of the form

$$
\begin{aligned}
& \dot{x}_{1}=A_{1} x_{1}(t)+B_{1} x_{2}(t)+C_{1} x_{1}(t-\tau)+D_{1} x_{2}(t-\tau), \\
& \dot{x}_{2}=A_{2} x_{1}(t)+B_{2} x_{2}(t)+C_{2} x_{1}(t-\tau)+D_{2} x_{2}(t-\tau),
\end{aligned}
$$

where $\tau>0, x_{1} \in R^{m_{1}}, x_{2} \in R^{m_{2}}, m_{1}+m_{2}=n, A_{i}, B_{i}, C_{i}, D_{i}, i=1,2$, are constant matrices of the corresponding dimensions.

Independent subsystems of system (5.1) are

$$
\begin{aligned}
& \dot{x}_{1}=A_{1} x_{1}(t)+C_{1} x_{1}(t-\tau), \\
& \dot{x}_{2}=B_{2} x_{2}(t)+D_{2} x_{2}(t-\tau) .
\end{aligned}
$$

For system (5.2) we construct the matrix-valued functional $U(t, \varphi)$ with the elements

$$
\begin{aligned}
& v_{11}=x_{1}^{T}(t) P_{11} x_{1}(t)+\int_{-\tau}^{0} x_{1}^{T}(t+s) P_{11} x_{1}(t+s) d s, \\
& v_{22}=x_{2}^{T}(t) P_{22} x_{2}(t)+\int_{-\tau}^{0} x_{2}^{T}(t+s) P_{22} x_{2}(t+s) d s, \\
& v_{12}=x_{1}^{T}(t) P_{12} x_{2}(t)+\int_{-\tau}^{0} x_{1}^{T}(t+s) P_{12} x_{2}(t+s) d s,
\end{aligned}
$$

which satisfy estimates characteristic for the quadratic forms 


$$
\begin{gathered}
\lambda_{m}\left(P_{11}\right)\left|x_{1}(t)\right|^{2}+\int_{-\tau}^{0} \lambda_{m}\left(P_{11}\right)\left|x_{1}(t+s)\right|^{2} d s \leq v_{11}\left(t, \varphi_{1}\right) \leq \\
\leq \lambda_{m}\left(P_{11}\right)\left|x_{1}(t)\right|^{2}+\int_{-r}^{0} \lambda_{M}\left(P_{11}\right)\left|x_{1}(t+s)\right|^{2} d s, \\
\lambda_{m}\left(P_{22}\right)\left|x_{2}(t)\right|^{2}+\int_{-\tau}^{0} \lambda_{m}\left(P_{22}\right)\left|x_{2}(t+s)\right|^{2} d s \leq v_{22}\left(t, \varphi_{2}\right) \leq \\
\leq \lambda_{M}\left(P_{22}\right)\left|x_{2}(t)\right|^{2}+\int_{-\tau}^{0} \lambda_{M}\left(P_{22}\right)\left|x_{2}(t+s)\right|^{2} d s, \\
-\lambda_{M}^{1 / 2}\left(P_{12} P_{12}^{T}\right)\left|x_{1}(t)\right|\left|x_{2}(t)\right|-\int_{-\tau}^{0} \lambda_{M}^{1 / 2}\left(P_{12} P_{12}^{T}\right)\left|x_{1}(t+s) \| x_{2}(t+s)\right| d s \leq \\
\leq v_{12}\left(t, \varphi_{1}, \varphi_{2}\right) \leq \lambda_{M}\left(P_{12} P_{12}^{T}\right)\left|x_{1}(t) \| x_{2}(t)\right|+ \\
+\int_{-\tau}^{0} \lambda_{M}\left(P_{12} P_{12}^{T}\right)\left|x_{1}(t+s) \| x_{2}(t+s)\right| d s .
\end{gathered}
$$

Here $P_{11}, P_{12}$ are symmetric positive definite matrices and $P_{12}$ is a constant matrix.

It is easy to verify that for the functional

$$
V(t, x, \eta)=\eta^{T} U(t, x(s)) \eta, \quad \eta=(1,1)^{T},
$$

the bilateral estimate

$$
\begin{gathered}
u_{0}^{T}(t) H^{T} A H u_{0}(t)+\int_{-\tau}^{0} u_{0}^{T}(t+s) H^{T} A H u_{0}(t+s) d s \leq V(t, x, \eta) \leq \\
\leq u_{0}^{T}(t) H^{T} B H u_{0}(t)+\int_{-\tau}^{0} u_{0}^{T}(t+s) H^{T} B H u_{0}(t+s) d s
\end{gathered}
$$

is valid, where

$$
\begin{gathered}
u_{0}^{T}(t)=\left(x_{1}^{T}(t), x_{2}^{T}(t)\right), \quad u_{0}^{T}(t+s)=\left(x_{1}^{T}(t+s), x_{2}^{T}(t+s)\right) \\
H^{T}=H=\operatorname{diag}(1,1), \\
A=\left(\begin{array}{cc}
\lambda_{m}\left(P_{11}\right) & -\lambda_{M}^{1 / 2}\left(P_{12} P_{12}^{T}\right) \\
-\lambda_{M}^{1 / 2}\left(P_{12} P_{12}^{T}\right) & \lambda_{m}\left(P_{22}\right)
\end{array}\right) \\
B=\left(\begin{array}{cc}
\lambda_{M}\left(P_{11}\right) & \lambda_{M}^{1 / 2}\left(P_{12} P_{12}^{T}\right) \\
\lambda_{M}^{1 / 2}\left(P_{12} P_{12}^{T}\right) & \lambda_{M}\left(P_{22}\right)
\end{array}\right) .
\end{gathered}
$$

It follows from estimate (5.6) that for functional (5.5) to be positive definite it is sufficient that the matrix $H^{T} A H$ to be positive definite.

For the upper right derivative of functional (5.5) along solutions of system (5.1) one can easily obtain the estimate

$$
\left.D^{+} V(t, x(s))\right|_{(5.1)} \leq u^{T} G u
$$

where the following notations are used 


$$
\begin{gathered}
u^{T}=\left(\left|u_{1}(t, \cdot)\right|,\left|u_{2}(t, \cdot)\right|\right), \\
G=\left(\begin{array}{cc}
\lambda_{M}(C)+2 \lambda_{M}(F) & \lambda_{M}^{1 / 2}\left(K K^{T}\right) \\
\lambda_{M}^{1 / 2}\left(K K^{T}\right) & \lambda_{M}(D)+2 \lambda_{M}(L)
\end{array}\right),
\end{gathered}
$$

where

$$
\begin{aligned}
& C=\left(\begin{array}{cc}
\lambda_{M}\left(A_{1}^{T} P_{11}+P_{11} A_{1}+P_{11}\right) & \lambda_{M}^{1 / 2}\left[\left(P_{11} C_{1}\right)\left(P_{11} C_{1}\right)^{T}\right] \\
\lambda_{M}^{1 / 2}\left[\left(P_{11} C_{1}\right)\left(P_{11} C_{1}\right)^{T}\right] & \lambda_{M}\left(-P_{11}\right)
\end{array}\right), \\
& D=\left(\begin{array}{cc}
\lambda_{M}\left(B_{2}^{T} P_{22}+P_{22} B_{2}+P_{22}\right) & \lambda_{M}^{1 / 2}\left[\left(P_{22} D_{2}\right)\left(P_{22} D_{2}\right)^{T}\right] \\
\lambda_{M}^{1 / 2}\left[\left(P_{22} D_{2}\right)\left(P_{22} D_{2}\right)^{T}\right] & \lambda_{M}\left(-P_{22}\right)
\end{array}\right) \text {, } \\
& F=\left(\begin{array}{cc}
\lambda_{M}\left(P_{12} A_{2}\right) & \frac{1}{2} \lambda_{M}^{1 / 2}\left(\left(P_{12} C_{2}\right)\left(P_{12} C_{2}\right)^{T}\right) \\
\frac{1}{2} \lambda_{M}^{1 / 2}\left(\left(P_{12} C_{2}\right)\left(P_{12} C_{2}\right)^{T}\right) & 0
\end{array}\right), \\
& K=\left(\begin{array}{cc}
\lambda_{M}^{1 / 2}\left(Q Q^{T}\right) & \lambda_{M}^{1 / 2}\left(Y Y^{T}\right) \\
\lambda_{M}^{1 / 2}\left(W W^{T}\right) & 0
\end{array}\right) \\
& L=\left(\begin{array}{cc}
\lambda_{M}\left(B_{1}^{T} P_{12}\right) & \lambda_{M}^{1 / 2}\left(\left(P_{12}^{T} D_{1}\right)\left(P_{12}^{T} D_{1}\right)^{T}\right) \\
\lambda_{M}^{1 / 2}\left(\left(P_{12}^{T} D_{1}\right)\left(P_{12}^{T} D_{1}\right)^{T}\right) & 0
\end{array}\right), \\
& Q=P_{11} B_{1}+A_{2} P_{22}^{T}+A_{1}^{T} P_{12}+P_{12} B_{2}, \\
& Y=P_{11} D_{1}+P_{12} D_{2} \text {, } \\
& W=C_{2}^{T} P_{22}+C_{1}^{T} P_{12} .
\end{aligned}
$$

Estimates (5.6) and (5.7) yield stability criterion for the state $x=0$ of system (5.1) formulated below.

Theorem 5.1. Assume that for system (5.1) the matrix-valued functional $U(t, \varphi)$ is constructed with components (5.3). If in estimate (5.6) the matrices $A$ and $B$ are positive definite and in estimate (5.7) the matrix $G$ is negative definite then the zero solution of system (5.1) is uniformly asymptotically stable.

The proof of this theorem follows from Theorem 4.1.

Remark 5.1. It is easy to show that conditions of Theorem 5.1 are fulfilled provided that

a) $\lambda_{M}\left(P_{11}\right) \lambda_{M}\left(P_{22}\right)>\lambda_{M}\left(P_{12} P_{12}^{T}\right)$,

b) $\lambda_{M}(C)+2 \lambda_{M}(F)<0$,

c) $\lambda_{M}(D)+2 \lambda_{M}(L)<0$,

d) $\left(\lambda_{M}(C)+2 \lambda_{M}(F)\right)\left(\lambda_{M}(D)+2 \lambda_{M}(L)\right)>\lambda_{M}\left(K K^{T}\right)$.

Further, to study system (5.1) we apply Approach $\mathrm{B}_{2}$ and the functional $U(\varphi)$ with the elements (5.3). Let $\eta \in R_{+}^{2}, \eta=(1,1)^{T}$. Then, in the framework of this approach one need to study sign-definiteness of the upper right derivative

$$
\begin{aligned}
\left.D^{+} V(x, \eta)\right|_{(5.1)} & =\left.D^{+} v_{11}\left(x_{1}\right)\right|_{(5.2)}+\left.D^{+} v_{22}\left(x_{2}\right)\right|_{(5.2)}+ \\
& +\left.2 D^{+} v_{12}\left(x_{1}, x_{2}\right)\right|_{(5.1)} .
\end{aligned}
$$


Having accomplished simple transformations in the expression (5.8) we get the estimate

$$
\left.D^{+} V(x, \eta)\right|_{(5.1)} \leq u^{T} S u,
$$

where $u^{T}=\left(\left|u_{1}\right|,\left|u_{2}\right|\right)$ and

$$
\begin{aligned}
& u_{1}^{T}=\left(\left|x_{1}(t)\right|,\left|x_{1}(t-\tau)\right|\right), \quad u_{2}^{T}=\left(\left|x_{2}(t)\right|,\left|x_{2}(t-\tau)\right|\right), \\
& S=\left(\begin{array}{cc}
\lambda_{M}^{1 / 2}(C) & \lambda_{M}^{1 / 2}\left(K K^{T}\right) \\
\lambda_{M}^{1 / 2}\left(K K^{T}\right) & \lambda_{M}^{1 / 2}(D)
\end{array}\right), \\
& C=\left(\begin{array}{ll}
c_{11} & c_{12} \\
c_{21} & c_{22}
\end{array}\right), \quad D=\left(\begin{array}{ll}
d_{11} & d_{12} \\
d_{21} & d_{22}
\end{array}\right), \quad K=\left(\begin{array}{ll}
k_{11} & k_{12} \\
k_{21} & k_{22}
\end{array}\right) \text {, } \\
& c_{11}=\lambda_{M}\left(A_{1}^{T} P_{11}+P_{11} A_{1}+P_{12} A_{2}+A_{2}^{T} P_{12}+P_{11}\right) \text {, } \\
& c_{21}=c_{12}=\lambda_{M}^{1 / 2}\left(\left(P_{11} C_{1}+P_{12} P_{2}\right)\left(P_{11} C_{1}+P_{12} C_{2}\right)^{T}\right) \text {, } \\
& c_{22}=\lambda_{M}\left(-P_{11}\right) \\
& d_{11}=\lambda_{M}\left(B_{2}^{T} P_{22}+P_{22} B_{2}+B_{1} P_{12}+P_{12}^{T} B_{1}^{T}+P_{22}\right) \text {, } \\
& d_{21}=d_{12}=\lambda_{M}^{1 / 2}\left(\left(P_{22} D_{2}+P_{12} D_{1}\right)\left(P_{22} D_{2}+P_{12} D_{1}\right)^{T}\right), \\
& d_{22}=\lambda_{M}\left(-P_{22}\right) \text {; } \\
& k_{11}=\lambda_{M}^{1 / 2}\left(\left(P_{11} B_{1}+A_{2}^{T} P_{22}+A_{1}^{T} P_{12}+P_{12} B_{2}+\frac{1}{2} P_{12}\right) \times\right. \\
& \left.\times\left(P_{11} B_{1}+A_{2}^{T} P_{22}+A_{1}^{T} P_{12}+P_{12} B_{2}+\frac{1}{2} P_{12}\right)^{T}\right), \\
& k_{12}=\lambda_{M}^{1 / 2}\left(\left(P_{11} D_{1}+P_{12} D_{2}\right)\left(P_{11} D_{1}+P_{12} D_{2}\right)^{T}\right) \text {, } \\
& k_{21}=\lambda_{M}^{1 / 2}\left(\left(P_{22} C_{2}+P_{12} C_{1}\right)\left(P_{22} C_{2}+P_{12} C_{1}\right)^{T}\right) \text {, } \\
& k_{22}=\frac{1}{2} \lambda_{M}^{1 / 2}\left(P_{12} P_{12}^{T}\right) .
\end{aligned}
$$

Estimates (4.6) and (5.9) provide the following result.

Theorem 5.2. Assume that for system (5.1) the matrix-valued functional $U(\varphi)$ is constructed with components (5.3). If in estimate (5.4) the matrices $A$ and $B$ are positive definite and in estimate (5.9) the matrix $S$ is negative definite, then the zero solution of system (5.1) is uniformly asymptotically stable.

Remark 5.2. It is easy to verify that conditions of Theorem 5.2 are satisfied provided that

a) $\lambda_{M}\left(P_{11}\right) \lambda_{M}\left(P_{22}\right)>\lambda_{M}\left(P_{12} P_{12}^{T}\right)$,

b) $\lambda_{M}(C)<0$,

c) $\lambda_{M}(D)<0$

d) $\lambda_{M}(C) \lambda_{M}(D)>\lambda_{M}\left(K K^{T}\right)$.

6. Bibliographical comments. The intensive investigation of functional differential equations is motivated by many problem from mechanics, biology, 
elasticity, replicator dynamics, viscoelasticity, electricity, reactor dynamics, heat flow, chemical oscillations, and neural networks. The books $[1,2,11,21]$ and papers $[3,13$, $20,22]$ are wonderful source of the problems in the direction.

In Section 2 we present some auxiliary results. It is based on some known results $[2,12,27]$.

In the Section 3 we used some results from $[15,18,19]$.

Many authors try to find effective approaches to construction of Liapunov functionals (see $[4-7,9,24,25]$ ). However the problem is, in general, open.

The approaches presented in this paper are aimed at solving the problem of stability of the zero solution of large-scale systems functional differential equations. These approaches have a considerable potential for further development and applications.

First, we note that insignificant modification of the conditions of Theorem 4.1 allows us to establish a new boundedness conditions for motions in functional differential systems with finite delay by further development of results of the papers $[8$, 10]. Also note that the proposed approach enables us to determine the stabilizing (destabilizing) role of delay, since it admits the existence of both stable and unstable subsystems in the initial system.

Second, the approach developed in this paper make it possible to apply efficiently some general results (see, for example, $[3,9,12]$ ) on stability of solutions to large-scale functional differential equations which contain functionals satisfying estimates of the type

$$
w_{1}(|\varphi(0)|) \leq v(t, \varphi) \leq w_{2}(|\varphi|)
$$

or its generalizations, for example, in the form

$$
w_{1}(|\varphi(0)|) \leq v(t, \varphi) \leq w_{2}(|\varphi|)+w_{3}\left(|\varphi| \mid \cdot \|_{L_{2}}\right)
$$

where $\|\cdot\|_{L_{2}}$ is the norm in space $L_{2}$, and $w_{i}(\cdot)$ is the comparison function of class $K(K R)$. We weaken conditions (6.1) or (6.2) by expansion of the set of components $v_{i j}$, appropriate for construction of suitable functional. Some possibilities of the proposed technique of stability analysis is applicable to quasilinear equations which remain an urgent object of investigations, including estimates of stability domains in parameter space.

1. Azbelev N. V., Simonov P. M. Stability of differential equations with aftereffect. - London: Taylor and Francis, 2002. - $222 \mathrm{p}$.

2. Burton T. A. Stability and periodic solutions of ordinary and functional differential equations. Orlando: Acad. Press, 1985.

3. Burton T. A., Hatvani L. Stability theorems for nonautonomous functional differential equations by Liapunov functionals // Tohoku Math. J. - 1989. - 41. - P. 65-104.

4. Dao Yi Xu. Uniform asymptotic stability in terms of two measures for functional differential equations // Nonlinear Anal. - 1996. - 27. - P. 413-427.

5. Driver R. D. Existence and stability of solutions of a delay-differential systems // Arch. Ration. Mech. and Anal. - 1962. - 10. - P. 401-426.

6. Infante E. F., Castelan W. B. A Liapunov functional for a matrix difference-differential equation // J. Different. Equat. - 1978. - 29. - P. 439-451.

7. Gaishun V. I., Knyazhishche L. B. Nonmonotone Lyapunov functionals. Stability conditions for delay differential equations // Differents. Uravn. - 1994. - 30, № 8. - P. 1291-1298.

8. Haddock J. R., Zhao J. Instability for autonomous and periodic functional differential equations with finite delay // Funkc. ekvacioj. - 1996. - 39. - P. 553-570.

9. Kim A. V. Direct Liapunov method in the stability theory of the systems with aftereffect (in Russian). - Ekaterinburg: Izd. Ural Univ., 1992. - 199 p.

10. Kobayashi K., Tsuruta $K$. Uniform boundedness and uniform asymptotic stability in functional differential equations with constant delay // Funkc. ekvacioj. - 1997. - 40. - P. 79-92.

11. Kolmanovskii V. B., Nosov V. R. Stability of functional differential equations. - London: Acad. Press, 1986.

12. Krasovskii N. N. Stability of motion. - Stanford: Stanford Univ. Press, 1963. - 297 p. 
13. Lakshmikantham V. Functional-differential systems and extension of Lyaponov's methods // J. Math. Anal. and Appl. - 1964. - 8. - P. 392-405.

14. Lakshmikantham V., Martynyuk A. A. Development of direct Liapunov method for the systems with delay // Prikl. Mekh. - 1993. - 29, № 2. - P. 3-16.

15. Martynyuk A. A. Matrix-valued functional approach for stability analysis of functional differential equations // Nonlinear Anal. - 2004. - 56. - P. 793-802.

16. Martynyuk A. A. Stability by Liapunov's matrix function method with applications. - New York: Marcel Dekker, 1998. - 276 p.

17. Martynyuk A. A. Qualitative methods in nonlinear dynamics: novel approaches to Liapunov's matrix functions. - New York: Marcel Dekker, 2002. - 301 p.

18. Martynyuk A. A., Rizaev R. On the applications of matrix-valued functionals in studying stability of system with delay // Dopov. Nat. Akad. Nauk Ukraine. - 2002. - № 2. - P. 15-19 (in Russian).

19. Martynyuk A. A., Rizaev R. Sufficient stability conditions for systems with delay // Int. Appl. Mech. - 2001. - 37, № 12. - P. 1612-1617.

20. Martynyuk A. A., Sun Zhenqi. The matrix-valued Liapunov functional and stability of systems with delay // Dokl. Math. - 1998. - 57, № 2. - P. 207-209.

21. Mitropolskii Yu. A., Martynyuk D. I. Lectures on the theory of vibrations of delay systems. - Kiev: Inst. Math. Acad. Sci. UkrSSR, 1969. - 309 p.

22. Ohta Y., Siljak D. D. An inclusion principle for hereditary systems // J. Math. Anal. and Appl.. 1984. - 98, № 2. - P. $581-598$.

23. Razumikhin B. S. The application of Lyapunov's method to problems in the stability of system with delay // Automat Remote Control. - 1960. - 21. - P. 515-520.

24. Repin I. M. Quadratic Liapunov functionals for systems with delays // Prikl. Mat. i Mech. - 1965. 29. - P. $564-566$.

25. Soo R. Lee, Jamshidi M. Stability analysis for large scale time delay systems via the matrix Lyapunov function // Kybernetika. - 1992. - 28. - P. 271-283.

26. Taniguchi T. Asymptotic behavior theorems for non-autonomous functional differential equations via Lyapunov - Razumikhin method // J. Math. Anal. and Appl. - 1995 - 189. - P. 715-730.

27. Zhang Bo. A stability theorem in functional differential equations // Different. and Integr. Equat. 1996. - 9. - P. 199-208.

Received 26.09.2006 